\title{
THE PROBLEM OF TIME IN PARAMETRIZED THEORIES*
}

\author{
Fabián H. GAIOLI and Edgardo T. GARCIA-ALVAREZ ${ }^{\dagger}$ \\ Departamento de Física, Facultad de Ciencias Exactas y Naturales, \\ Universidad de Buenos Aires, 1428 Buenos Aires, Argentina \\ 8 \\ Instituto de Astronomía y Física del Espacio, \\ C.C. 67, Suc. 28, 1428 Buenos Aires, Argentina
}

(October 13, 2018)

\begin{abstract}
A common feature of reparametrization invariant theories is the difficulty involved in identifying an appropriate evolution parameter and in constructing a Hilbert space on states. Two well known examples of such theories are the relativistic point particle and the canonical formulation of quantum gravity. The strong analogy between them (specially for minisuperspace models) is considered in order to stress the correspondence between the "localization problem" and the "problem of time," respectively. A possible solution for the first problem was given by the proper time formulation of relativistic quantum mechanics. Thus, we extrapolate the main outlines of such a formalism to the quantum gravity framework. As a consequence, a proposal to solve the problem of time arises.
\end{abstract}

Pacs numbers: 03.65.Pm, 04.60.+n

*Published in General Relativity and Gravitation 26, 1267-1275 (1994)

†E-mail: gaioli@iafe.uba.ar; galvarez@dfuba.df.uba.ar 
Since B.S. DeWitt's celebrated paper [1] about the canonical formulation of quantum gravity (QG) was published, the problem of time and the interpretation of the wave functional have remained unsolved.

The main difficulty in reconciling the antagonistic formalisms of quantum mechanics and the theory of relativity lies in the role and interpretation of "time." This is true not only in the case of general relativity, but also at the level of the special theory of relativity. In fact, while the latter theory deals with the space-time coordinates on an equal footing, quantum mechanics privileges an external absolute parameter which labels the evolution of the state of the system. Therefore, in the second case, "time" should have the properties of a $c$-number, unlike in the first case where, since the spatial coordinates are raised to the status of operators, Lorentz transformations should impose this character on the temporal coordinate as well. Thus, this dual role of "time" generates a conflict in relativistic quantum mechanics (RQM). This is basically the reason why a one-particle theory in the usual formulation of RQM has several conceptual difficulties, such as the interpretation of negative energies which gave origin to the famous "problem of localization." (An exhaustive review of the localization problem up to the $70 \mathrm{~s}$ can be found in [2]).

The history of physics teaches us that every conflict arising from the unification of two fundamental theories reveals the incompatibility among hypotheses of the two theories. Thus, some of them must be removed and therefore some prejudices we firmly cling to must be overcome.

In the 40s a proper time formalism (PTF) was developed [3] for a possible unification between special relativity and quantum mechanics, within the framework of a consistent one-particle theory. However, the price that we must pay to celebrate this wedding is to give up the concept of definite mass state.

The PTF introduces an evolution absolute parameter $\tau$, independent of the proper time but related to it through the classical limit [4], which parametrizes the dynamics of the system. This parameter plays the role of "time" [5.,6], while the temporal coordinate has a different status, being promoted to the rank of operator. The classical mass constraint is removed but nevertheless the standard RQM is recovered on-shell. In this way the formalism closely copies the general outlines of the nonrelativistic quantum mechanics, furnishing the theory with a well known structure.

This matter has recently gained renewed interest with the appearance of several works that intended to go beyond the formal aspects. (Versions of RQM similar to the one presented here have been considered, e.g., in Refs. [3,5,6]. See also Refs. [7,8]).

In the last decade the analogy between the canonical formulation of QG and the spinless relativistic point particle has been widely exploited [9]. Therefore, it is not surprising to find the same kind of difficulties since both systems are similar in nature. These issues are known in QG as "the problem of time" [10].
The aim of this essay is two-fold. On the one hand we try to connect two lines of research, the localization problem in RQM and the problem of time in QG. On the other hand, we propose a parametrized theory for QG, which can be considered as a promising solution to the problem of time.

For the sake of simplicity, in this essay we only consider minisuperspace models for QG 11]. The full superspace formalism will be discussed elsewhere [12].

Let us start with the spinless relativistic point particle in the PTF. Let $\left\{\left|x^{\mu}\right\rangle\right\} \quad(\mu=0,1,2,3)$ be the basis of localized states of the position operator $X^{\mu}$ spanning a linear space endowed with a scalar product,

$$
\langle\Phi \mid \Psi\rangle=\int d^{4} x \Phi^{*}\left(x^{\mu}\right) \Psi\left(x^{\mu}\right),
$$

satisfying the normalization and the completeness conditions,

$$
\left\langle x^{\mu} \mid x^{\prime \mu}\right\rangle=\delta\left(x^{\mu}-x^{\prime \mu}\right), \quad \int d^{4} x\left|x^{\mu}\right\rangle\left\langle x^{\mu}\right|=\mathbf{I} .
$$

In this coordinate representation the state of the system is represented by the wave function, belonging to a fourdimensional Hilbert space,

$$
\Psi\left(x^{\mu}\right)=\left\langle x^{\mu} \mid \Psi\right\rangle,
$$

defined on the space-time manifold. The position operator and its canonical conjugate variable, the momentum $P_{\mu}$, satisfying $(\hbar=c=1)$

$$
\left[X^{\mu}, P^{\nu}\right]=-i \eta^{\mu \nu}, \quad\left(\eta^{\mu \nu}=\operatorname{diag}\{+1,-1,-1,-1\}\right),
$$

adopt the well known expressions,

$$
\begin{gathered}
\left\langle x^{\mu}\left|X^{\mu}\right| \Psi\right\rangle=x^{\mu} \Psi\left(x^{\mu}\right), \\
\left\langle x^{\mu}\left|P_{\mu}\right| \Psi\right\rangle=i \frac{\partial}{\partial x^{\mu}} \Psi\left(x^{\mu}\right) .
\end{gathered}
$$

(In Eq. (何) $-i$ was chosen to preserve the sign in the ordinary relations for the spatial part.)

In the Schrödinger picture,

$$
\left|\Psi\left(x^{\mu}, \tau\right)\right|^{2},
$$

represents the probability density for the system to be at the space-time point $x^{\mu}$ at "instant" $\tau$. The wave function evolves with a Schrödinger equation,

$$
-i \frac{d}{d \tau} \Psi\left(x^{\mu}, \tau\right)=\mathcal{H} \Psi\left(x^{\mu}, \tau\right),
$$

where

$$
\mathcal{H}=\frac{1}{2} \eta^{\mu \nu} P_{\mu} P_{\nu}
$$


is a super-Hamiltonian (free case) which plays the role of a mass operator [13.

The super-Hamiltonian as well as position and momentum operators are Hermitian in the inner product (1).

A stationary solution of Eq. (7) is

$$
\Psi\left(x^{\mu}, \tau\right)=e^{i \mathcal{H} \tau} \psi\left(x^{\mu}\right),
$$

where $\psi\left(x^{\mu}\right)$ is a solution of a generalized Klein-Gordon equation,

$$
\frac{\partial}{\partial x^{\mu}} \frac{\partial}{\partial x_{\mu}} \psi\left(x^{\mu}\right)+m^{2} \psi\left(x^{\mu}\right)=0,
$$

where the mass eigenvalue $m^{2}$ is not restricted a priori to be positive. Thus, the theory will describe real (onshell) and virtual (off-shell) particles in a more symmetric way, which could be appropriate for the curved spacetime case, where the notion of real and virtual particles is relative.

We can see that the theory developed is formally identical to ordinary quantum mechanics. Then, all we have learned from this theory can be rewritten in the PTF.

The PTF can be formally obtained, via a canonical quantization procedure, from an indefinite mass classical theory. It corresponds to a unitary realization of a symmetry group which, at the classical level, is given by a subgroup of canonical transformations, whose generating function reads

$$
G_{X P M}=-\frac{\epsilon_{\mu \nu}}{2} M^{\mu \nu}+a^{\mu} P_{\mu}+b_{\mu} X^{\mu},
$$

where $\epsilon_{\mu \nu}=-\epsilon_{\nu \mu}, a^{\mu}$, and $b_{\mu}$ are real parameters. (Homogeneous Lorentz transformations are represented by the unitary operator $\mathcal{L}=\exp \left\{-\frac{i}{2} \epsilon_{\mu \nu} M^{\mu \nu}\right\}$, under the scalar product given in (11).)

The algebra of the generators of such a group, $X^{\mu}, P_{\mu}$, and $M^{\mu \nu}=X^{\mu} P^{\nu}-X^{\nu} P^{\mu}+\frac{1}{2} \sigma^{\mu \nu}\left(\sigma^{\mu \nu}\right.$ is the spin tensor), is known as $X P M$ algebra [8]. The irreducible representations of the $X P M$ group are constructed from the direct product of a covariant version of the HeisenbergWeyl group and the homogeneous Lorentz group. In the spin 0 case discussed above, this enlarged Poincaré group can be represented in the wave function space defined in Eq. (3).

Let us now come back to the localization problem. Note that from Eq. (4) it is immediate that, in general,

$$
\left[\mathcal{H}, X^{\mu}\right] \neq 0
$$

while the commutator vanishes in the standard (on-shell) RQM, showing that the Heisenberg algebra (4) is only realizable in an indefinite mass theory. Moreover, from (12) we can readily see that it is not possible to localize definite mass states preserving the Lorentz invariance of localization. Position operators such as the NewtonWigner one [14 are recovered on-shell, but now their interpretation can be clarified (Ref. [15], and see also Ref. [5]).
The localization problem lies in the fact that the temporal coordinate has an ambiguous character in RQM. Such a problem could be actually called "the problem of time in RQM" - at least for the usual notion of time. As a consequence it is not possible, in this scheme, to find a consistent quantum mechanical formulation for the relativistic particle (i.e., the construction of a Hilbert space with a conserved non-negative norm). This issue can be bypassed in two alternative ways. One can consider the temporal coordinate as a parameter, but then the oneparticle interpretation fails. Thus, one can abandon this avenue and turn to quantum field theory, where spacetime coordinates are parameters. This way does not solve the localization problem; the problem was swept under the rug [16]. On the other hand, using the PTF one can return to a one-particle theory (completely equivalent to quantum field theory but with the significant advantage that one can deal with a "one-particle" configuration space; Ref. [17]). Introducing an evolution absolute parameter $\tau$, the space-time coordinates can now acquire the same status as operators.

PTF "solves" the problem of time in RQM. However, we have learned something else: the way to construct a consistent quantum formalism in an originally reparametrization invariant theory. This is the important analogy that we can mimic in QG. Of course, there is no problem with the role played in QG by time if we do not adopt the canonical quantization procedure [18. 2 If we adopt this proceduce, the problem then is that time is completely absent in the Wheeler-DeWitt equation. What is still unsolved is the construction of a suitable quantum mechanical theory for a wave function independent of time. The PTF provides a way.

Let us compare the relativistic particle with the canonical formulation of QG for minisuperspace models.

The analog of the super-Hamiltonian (8) is given (in the $3+1$ decomposition of the metric) by

$$
\mathcal{H}_{\mathrm{m}}=\frac{1}{2 M^{2}} f^{a b}(q) p_{a} p_{b}+M^{2} V(q)
$$

which resembles the Hamiltonian of a particle of "mass" $M^{2}$ in a curved background. $f_{a b}$ is a metric on minisuperspace and $M$ is the Planck mass (the subscript $\mathrm{m}$ denotes minisuperspace). $p_{a}$ is the momentum conjugate to $q^{a}$ which represents certain components of the threemetric and certain modes of the matter fields. The term $V(q)$, which plays the role of a potential, could also be introduced for the relativistic particle but it does not modify the line of this discussion.

\footnotetext{
${ }^{1}$ The usual on-shell covariant action for a spinless particle is invariant under reparametrizations of the "label time" (which is not the parameter $\tau$ of our proposal).

${ }^{2}$ In the context of minisuperspace models one can overcome the problem of time by adopting a "third" quantization theory. See e.g., Ref. [19].
} 
We can establish a paralellism with the formalism developed above. Considering the configuration variables $q^{a}$, we can repeat the reasonings of Eqs. (1)-(6), defining the inner product (conserved in $\tau$ ) as

$$
\langle\Phi \mid \Psi\rangle=\int d^{n} q \sqrt{-f} \Phi^{*}\left(q^{a}\right) \Psi\left(q^{a}\right)
$$

where $n$ is the number of minisuperspace coordinates, and identifying

$$
X^{\mu} \longrightarrow q^{a}, \quad P_{\mu} \longrightarrow p_{a},
$$

as canonical variables. Thus, we can give a Hilbert space structure to the space of wave functions $\Psi\left(q^{a}\right)$ by demanding that they be square integrable with respect to $q^{a}$. The corresponding evolution equation reads

$$
-i \frac{d}{d \tau} \Psi\left(q^{a}, \tau\right)=\mathcal{H}_{\mathrm{m}} \Psi\left(q^{a}, \tau\right)
$$

where $\mathcal{H}_{\mathrm{m}}$ is given by Eq. (13). (The time-dependent Wheeler-DeWitt equation has also been discussed recently in Refs. 20,21].)

The rest of the formalism follows the outlines of quantum mechanics. Obviously, in this proposal we are not forced to restrict the allowed "observables" in the theory to be those which commute with the Hamiltonian (as it happens in the "naive interpretation" of the WheelerDeWitt equation, Ref. [21). Nevertheless, as in Eq. (10), we can restrict the theory to admit only the on-shell solutions of the eigenvalue 0 , which correspond to solutions of the Wheeler-DeWitt equation.

The retarded propagator of the Schrödinger equation (16), which gives the transition amplitude for the system "to evolve" between $q_{0}^{a}$ at $\tau=0$ and $q^{a}$ at $\tau$, is just $\theta(\tau)\left\langle q^{a}\left|e^{i \mathcal{H}_{\mathrm{m}} \tau}\right| q_{0}^{a}\right\rangle$. The corresponding time independent Green function reads

$$
G\left(q^{a}, q_{0}^{a}\right)=\int_{0}^{+\infty} d \tau\left\langle q^{a}\left|e^{i \mathcal{H}_{\mathrm{m}} \tau}\right| q_{0}^{a}\right\rangle
$$

In the analogous expression for the relativistic particle, selecting a particular value of the mass and putting a regulator $+i 0$ term in order to damp the oscillations for large $\tau$, one obtains the standard Green function for the Klein-Gordon equation, with the Feynman prescription to avoid the poles. This is just the result obtained from the second quantization of the Klein-Gordon field. In the same way the Green function (17) is equal to those derived by proceeding to the quantum field theory of the Universe ("third quantization").

The propagator in Eq. (17) can be conveniently expressed via a path integral. The derivation essentially follows the same steps as in ordinary quantum mechanics (see, e.g., Ref. [22]; the original derivation of the path integral of a particle in curved space-time can be found in Ref. [23]). It gives
$G\left(q^{a}, q_{0}^{a}\right)=\int_{0}^{\infty} d \tau \int \mathcal{D} q \mathcal{D} p \exp \left\{-i \int_{0}^{\tau}\left[p_{a} \dot{q}^{a}-\mathcal{H}_{\mathrm{m}}\right] d \tau^{\prime}\right\}$

where we can see the classical action $S=\int_{0}^{\tau}\left[p_{a} \dot{q}^{a}-\right.$ $\left.\mathcal{H}_{\mathrm{m}}\right] d \tau^{\prime}$ corresponding to the "free constraint" theory. These calculations are examples of a very important property of the PTF. Since in the theory, the constraint $\mathcal{H}_{\mathrm{m}}=0$ is not present, the path integral does not present any difficulty, unlike the constrained system. The same result (18) has been recently obtained by Halliwell [24] using the method introduced by Batalin, Fradkin, and Vilkovisky [25] based on the BRST invariance. To compare his result with ours we must identify $d \tau$ with $d N t$, where $N$ is the lapse function appearing as a Lagrange multiplier in the action $S=\int_{0}^{t}\left[p_{a} \dot{q}^{a}-N \mathcal{H}_{\mathrm{m}}\right] d t^{\prime}$. The latter identification was originally made by Teitelboim 26] for the relativistic particle as well as the gravitational field, imposing a gauge fixing condition. Even if the different approaches give the same expression for the Feynman Green function, we believe that our proposal is not only a very simple way to attack the problem of time but also gains in physical content. 3

The original goal of this essay was to establish a paralellism between the localization and the time problems. However, a door is open: the off-shell theory (such as the virtual particles appearing in the PTF of RQM). Its physical implications and a more detailed discussion will be considered in a separate work [12].

\section{ACKNOWLEDGMENTS}

This essay received an Honorable Mention in the 1994 Awards for Essays on Gravitation of the Gravity Research Foundation, which we gratefully acknowledge.

We would like to thank Claudia Bernardou, Luca Bombelli, Mario Castagnino, Graciela Garcia Alvarez, and Sebastiano Sonego for their help. One of us (F.H.G.) wishes to thank Edgard Gunzig and Ilya Prigogine for hospitality at the Université Libre de Bruxelles when this work was carried out.

\footnotetext{
${ }^{3}$ As was pointed out by Stephens 17 there are several different approaches by which one may arrive at the PTF for the relativistic particle and the same statement is valid for QG. From these alternatives we have chosen the Feynman proposal which has a richer physical content than the others.
} 
[1] B.S. DeWitt, Phys. Rev. 160, 1113 (1967).

[2] A.J. Kálnay, in Problems in the Foundations of Physics, ed. M. Bunge (Springer-Verlag, Berlin, 1971).

[3] V. Fock, Phys. Z. Sowjetunion 12, 404 (1937); E.C.G. Stückelberg, Helv. Phys. Acta 14, 322 (1941); 14, 558 (1941); 15, 23 (1942); R.P. Feynman, Phys. Rev. 80, 440 (1950); 84, 108 (1951). A review of Feynman work can be found in S.S. Schweber, Rev. Mod. Phys. 58, 449 (1986); Y. Nambu, Prog. Theor. Phys. 5, 82 (1950); J. Schwinger, Phys. Rev. 82, 664 (1951).

[4] J.P. Aparicio, F.H. Gaioli, and E.T. Garcia-Alvarez, "Interpretation of the evolution parameter of the Feynman parametrization of the Dirac equation," submitted to Phys. Lett. A; J.P. Aparicio, F.H. Gaioli, and E.T. Garcia-Alvarez, "Proper time formulation of relativistic quantum mechanics," to appear in Anales AFA 4.

[5] L.P. Horwitz and C. Piron, Helv. Phys. Acta 46, 316 (1973); M.C. Land, N. Shnerb, and L.P. Horwitz, "Feynman's proof of the Maxwell equations as a way beyond the standard model," Preprint TAUP-2076-93, and references quoted therein.

[6] S. Sonego, Phys. Rev. A 44, 5369 (1991).

[7] J.H. Cooke, Phys. Rev. 166, 1293 (1968); R.E. Collins and J.R. Fanchi, Nuovo Cimento A 48, 314 (1978); L. Hostler, J. Math. Phys. 21, 2461 (1980); A. Kyprianidis, Phys. Rep. 155, 2 (1987) and references cited therein.

[8] J.E. Johnson, Phys. Rev. 181, 1755 (1969); Phys. Rev. D 3, 1735 (1971).

[9] M. Henneaux and C. Teitelboim, Ann. Phys. (NY) 143, 127 (1982); J.B. Hartle and K.V. Kuchař, Phys. Rev. D 34, 2323 (1986).

[10] C. Isham, "Canonical quantum gravity and the problem of time," Imperial College preprint TP/91-92/25, grqc/9210011; K.V. Kuchař, in Proc. $4^{\text {th }}$ Canadian Conference on General Relativity and Relativistic Astrophysics, eds. G. Kunstatter, D. Vincent, and J. Williams (World Scientific, Singapore, 1992).

[11] J.J. Halliwell, in Proc. Jerusalem Winter School for Theoretical Physics: Quantum Cosmology and Baby Universe, vol. 7, eds. S. Coleman, J.B. Hartle, T. Piran, and S. Weinberg (World Scientific, Singapore, 1991).

[12] M.A. Castagnino, F.H. Gaioli, and E.T. Garcia-Alvarez, "Parametrized quantum gravity," in preparation.

[13] H. Enatsu, Prog. Theor. Phys. 30, 236 (1963); H. Enatsu and S. Kawaguchi, Nuovo Cimento 27A, 458 (1975).

[14] T.D. Newton and E.P. Wigner, Rev. Mod. Phys. 21, 400 (1949).

[15] P. Roman and J.P. Leveille, J. Math. Phys. 15, 2053 (1974).

[16] A.S. Wightman and S.S. Schweber, Phys. Rev. 98, 812 (1955); A.O. Barut and S. Malin, Rev. Mod. Phys. 40, 632 (1968). See also D. Lurié, Particles and Fields (Interscience Publishers, Bristol, 1968).

[17] C.R. Stephens, Ann. Phys. (NY) 181, 120 (1988).

[18] B.S. DeWitt, Phys. Rev. 162, 1195; 1239 (1967).

[19] A. Hosoya and M. Morikawa, Phys. Rev. D 39, 1123 (1989).

[20] J.D. Brown and J.W. York, Jr., Phys. Rev. D 40, 3312 (1989); M. Henneaux and C. Teitelboim, Phys. Lett. B 222, 195 (1989).
[21] W.G. Unruh and R.M. Wald, Phys. Rev. D 40, 2598 (1989).

[22] P. Ramond, Field Theory (Benjamin, Massachusetts, 1981).

[23] B.S. DeWitt, Rev. Mod. Phys. 29, 1 (1957). See also K.S. Cheng, J. Math. Phys. 13, 1723 (1972); L. Parker, Phys. Rev. D 19, 438 (1979).

[24] J.J. Halliwell, Phys. Rev. D 38, 2468 (1988). See also J. Guven and M.P. Ryan, Jr., Phys. Rev. D 45, 3559 (1992).

[25] E.S. Fradkin and G.A. Vilkovisky, Phys. Lett. B 55, 2 (1975); I.A. Batalin and G.A. Vilkovisky, ibid 69, 309 (1977). See also M. Henneaux, Phys. Rep. 126, 1 (1985).

[26] C. Teitelboim, Phys. Lett. B 96, 77 (1980); Phys. Rev. D 25, 3159 (1982); 28, 297 (1983); 28, 310 (1983). 